\title{
Conhecimento nutricional entre mulheres Obesas e Eutróficas atendidas em uma unidade ambulatorial de saúde: Classificaç̧ão e Comparação entre os Grupos
}

Nutritional knowledge among Obese and Eutrophic women treated at an outpatient health unit: Classification and
Comparison between Groups Conocimiento nutricional entre mujeres obesas y eutróficas atendidas en una unidad de salud ambulatoria: clasificación y comparación entre grupos

\begin{abstract}
RESUMO
Objetivo: Comparar o nível de conhecimento nutricional entre mulheres obesas e eutróficas. Métodos: Estudo transversal, amostra não probabilística de mulheres obesas (n 64) e eutróficas (n 64), idade entre 19 e 60 anos. Coletou-se dados sociodemográficos, estilo de vida, antropométricos (IMC, CC), QCN e Questionário Internacional de Atividade Física (IPAQ), versão curta. Resultados: Nível de conhecimento nutricional apresentado pela maioria de "moderado" (72\% MO e 82,8\% ME). Classificação de atividade física, a maioria das participantes de ambos os grupos se apresentaram "ativo" (59,4\% MO e 70,3\% ME). Quando associado o NCN e as variáveis sociodemográficas, percebeu-se associação positiva entre o nível de conhecimento nutricional "moderado/alto" de ambos os grupos; renda familiar 1 a 2 salários mínimos $(p=0,023)$; estilo de vida, etilismo ( $p=0,016)$ e atividade física classificados como "ativos". Quanto às associações entre as variáveis antropométricas (CC e IMC), não houve associação estatisticamente significante entre os índices. Conclusão: A maioria do grupo de obesas e eutróficas apresentou um nível de conhecimento nutricional "moderado", sem diferenças entre os grupos, sendo este associado a maior renda familiar, maior consumo de bebida alcoólica e indivíduos mais ativos.
\end{abstract}

DESCRITORES: Conhecimento, Estado nutricional, Obesidade.

\section{ABSTRACT}

Objective:To compare the level of nutritional knowledge between obese and eutrophic women. Methods: Cross-sectional study, non-probabilistic sample of obese (n 64) and eutrophic (n 64) women, aged between 19 and 60 years. Sociodemographic, lifestyle, anthropometric data (BMI, WC), QCN and International Physical Activity Questionnaire (IPAQ), short version, were collected. Results: Level of nutritional knowledge presented by the majority of "moderate" (72\% OM and 82.8\% ME). Classification of physical activity, the majority of participants in both groups were "active" (59.4\% OM and 70.3\% ME). When the NCN and the sociodemographic variables were associated, a positive association was perceived between the level of "moderate / high" nutritional knowledge of both groups; family income 1 to 2 minimum wages ( $p=0.023$ ); lifestyle, alcoholism ( $p=0.016)$ and physical activity classified as "active". As for the associations between anthropometric variables (WC and BMI), there was no statistically significant association between the índices. Conclusion: The majority of the obese and eutrophic group had a "moderate" level of nutritional knowledge, with no differences between the groups, which was associated with higher family income, greater consumption of alcoholic beverages and more active individuals.

DESCRIPTORS: Knowledge, Nutritional status, Obesity.

\section{RESUMEN}

Objetivo: Comparar el nivel de conocimiento nutricional entre mujeres obesas y eutróficas. Métodos: Estudio transversal, muestra no probabilística de mujeres obesas (n 64) y eutróficas (n 64), con edades comprendidas entre 19 y 60 años. Se recogieron datos sociodemográficos, de estilo de vida, antropométricos (IMC, CC), QCN y Cuestionario Internacional de Actividad Física (IPAQ), versión corta. Resultados: Nivel de conocimiento nutricional presentado por la mayoría de "moderados" (72\% OM y 82,8\% EM). Clasificación de la actividad física, la mayoría de los participantes en ambos grupos eran "activos" (59,4\% OM y 70,3\% ME). Cuando se asociaron la NCN y las variables sociodemográficas, se percibió una asociación positiva entre el nivel de conocimiento nutricional "moderado / alto" de ambos grupos; renta familiar 1 a 2 salarios mínimos ( $p=0,023$ ); estilo de vida, alcoholismo ( $p$ = 0,016) y actividad física clasificada como "activa". En cuanto a las asociaciones entre variables antropométricas (CC e IMC), no hubo asociación estadísticamente significativa entre los índices. Conclusión: La mayoría del grupo obeso y eutrófico tenía un 
nivel "moderado" de conocimiento nutricional, sin diferencias entre los grupos, lo que se asoció con mayores ingresos familiares, mayor consumo de bebidas alcohólicas e individuos más activos.

DESCRIPTORES: Conocimiento, Estado nutricional, Obesidad.

RECEBIDO EM: 30/07/2020 APROVADO EM: 21/10/2020

\section{Matheus Sobral Silveira}

Docente do curso de Nutrição no Centro Universitário Estácio da Bahia. Mestre em Medicina e Saúde Humana, Pós graduado em Nutrição Clínica/ Terapia Nutricional e Fitoterapia Clínica. Nutricionista (ESTÁCIO/FIB).

ORCID: 0000-0003-2005-2927

\section{Tatiane Melo de Oliveira}

Doutoranda em Gerontologia (UNICAMP/SP), Mestre em Alimentos, Nutrição e Saúde (UFBA), Especialista em Nutrição Clínica sob a forma de residência (UFBA/SESAB). Nutricionista (UFBA).

ORCID: 0000-0001-7938-9351

\section{Adriele Santos de Oliveira}

Mestra em Nutrição Clínica pela Universidade Católica San Antonio de MurciaV (UCAM) Espanha. Nutricionista (UFBA).

ORCID: 0000-0002-5057-143X

\section{Denise Terezinha Santana Santos}

Nutricionista. Pós-graduanda em Vigilância Sanitária e Qualidade de Alimentos (ESTÁCIO). Nutricionista (UCSAL).

ORCID: 000-0001-7265-4722

\section{Roberta Ribeiro de Menezes}

Nutricionista do Grupo de Pesquisa Promoção da Saúde - Pró Saúde. Nutricionista (UCSAL)

ORCID: 0000-0003-1108-9218

\section{Maria de Lourdes Lima}

Professora Adjunto na Pós graduação da Escola Bahiana de Medicina e Saúde Pública. Doutora e Mestre em Medicina e Saúde (UFBA), Especialista em Endocrinologia sob a forma de residência (UFBA). Médica Endocrinologista.

ORCID: 0000-0002-2081-4162

\section{INTRODUÇÃO}

$\longrightarrow$ onhecer sobre uma alimentação adequada e equilibrada é uma etapa importante para conscientizar-se que toda substância que ingerimos vai reagir de forma específica no nosso corpo. Ter essa consciência pode ser o primeiro passo para mudanças em comportamento alimentar inadequado. $\mathrm{O}$ comer é um ato biológico necessário para sobreviver, mas a alimentar-se vai além do nutrir-se. Envolve aspectos emocionais, culturais e sociais que transformam a simples ação biológica de comer. ${ }^{1}$

A nutrição busca o equilíbrio entre a alimentação e a saúde do corpo visando melhor qualidade de vida. $\mathrm{O}$ desequilíbrio alimentar pode ser um dos fatores etio- lógicos de doenças como obesidade que hoje é considerada um grande problema de saúde pública. $\mathrm{O}$ aumento da ingestão calórica que está envolvido no excesso de peso, ocorre principalmente as custas do consumo excessivo de alimentos processados que, muitas vezes são escolhidos por serem opções rápidas e práticas ${ }^{1,2}$.

Muitos fatores, extra alimentares podem influenciar o nível de conhecimento nutricional e o comportamento alimentar, sendo os mais citados pelos estudiosos do assunto o gênero, nível socioeconômico, cultura, etnia e escolaridade. Nas mulheres, por exemplo, quanto maior a escolaridade menor são as taxas de sobrepeso e obesidade observadas. Em contrapartida, para os homens quanto mais anos de estu- do, mais elevados são as taxas de sobrepeso e obesidade ${ }^{2,3}$.

Nas últimas décadas ocorreu um aumento importante do interesse sobre a melhor forma de alimentar-se e consequentemente a busca pelo conhecimento do que seja uma alimentação mais saudável também aumentou. Existem diversos métodos para avaliação da ingestão alimentar do indivíduo, porém pensando nos aspectos subjetivos, o Questionário de Conhecimento Nutricional (QCN) vem sendo postulado como uma ferramenta conceituada. O QCN representa um constructo científico criado por educadores nutricionais objetivando representar o processo cognitivo individual relacionado à informação sobre alimentação e nutrição ${ }^{4,5}$. 
A contribuição do conhecimento nutricional para a qualidade global da ingestão alimentar é considerada complexa e influenciada pela interação de fatores internos e do ambiente. No entanto, melhorar a compreensão da relação entre conhecimento nutricional, ingestão alimentar e estado nutricional é relevante como elo para o desenvolvimento de políticas preventivas contra as doenças crônicas não transmissíveis (DCNTs), principalmente por estas serem destaque entre as causas representativas de óbitos na população brasileira. A obesidade e suas consequências fazem parte do panorama epidemiológico de doenças incapacitantes, tornando-se, portanto, um desafio grande para as políticas públicas de saúde ${ }^{3,6}$.

O nutricionista tem papel fundamental na realização de promoção de saúde, focando em intervenções direcionadas para educação nutricional (cursos de capacitação, oficinas, consultas, palestras, grupos de apoio, entre outros). Todas essas ferramentas são conduzidas com o intuito de aumentar o conhecimento sobre nutrição nas pessoas, melhorando assim os seus hábitos alimentares e consequentemente prevenindo doenças.

Infelizmente a atuação do nutricionista nas unidades básicas de saúde ainda é insuficiente, o que acaba reduzindo o acesso da população aos conhecimentos associados à este profisisonal ${ }^{6,7}$. Tendo em vista o exposto, o presente trabalho classificou o nível de conhecimento nutricional de mulheres obesas e eutróficas, atendidas em uma unidade ambulatorial de saúde e realizou comparaçôes entre os grupos.

\section{MÉTODOS}

Estudo de corte transversal e analítico, realizado nos ambulatórios de excesso de peso e ginecologia do Centro Médico da Bahiana pertencente à Escola Bahiana de Medicina e Saúde Pública (EBMSP), em Salvador, BA, nos meses de setembro 2016 a abril de 2017. Aprovado pelo Comitê de Ética em Pesquisa (CEP) da Fundação Bahiana para Desenvolvimento das Ciências FBDC da EBMSP em agosto de 2016, sob o parecer de número 1694789.
O nutricionista tem papel fundamental na realização de promoção de

saúde, focando

em intervenções

direcionadas para educação nutricional (cursos de capacitação, oficinas, consultas, palestras, grupos de apoio, entre outros). Todas

essas ferramentas são conduzidas com o intuito de aumentar

o conhecimento

sobre nutrição nas

pessoas, melhorando

assim os seus hábitos

alimentares e

consequentemente

prevenindo doenças.
Após assinatura do Termo de Consentimento Livre e Esclarecido (TCLE) foram incluídas neste estudo mulheres com idade entre 19 a 63 anos.

Não foram incluídas mulheres com índice de massa corporal (IMC) fora da faixa de obesidade ou eutrofia; amputadas ou com dificuldades para deambular; gestantes ou lactantes; com escolaridade inferior ao quinto ano do ensino fundamental; comprometimento cognitivo que impedisse responder os questionários aplicados ou que receberam algum tipo de orientação/ acompanhamento com nutricionista nos últimos doze meses anterior a coleta dos dados.

Foram coletados dados sociais (idade, raça autorreferida, escolaridade, renda familiar, ocupação, e estado civil) por meio de questionário desenvolvido para este estudo. Para avaliação clínica foram coletadas informações sobre presença ou ausência de doenças e de estilo de vida (ingestão de bebida alcoólica, tabagismo, nível de atividade física). Para avaliação de estado nutricional foram coletados dados de peso, altura, circunferência da cintura, IMC. A altura foi aferida com um estadiômetro fixo. $\mathrm{O}$ peso foi aferido em balança digital portátil, da marca Filizola. O IMC foi calculado a partir dos dados coletados.

A avaliação da circunferência da cintura (CC) foi realizada através de fita métrica inelástica, com precisão de $1,0 \mathrm{~mm}$ (TBW, São Paulo, Brasil), no ponto médio entre a última costela flutuante e a crista ilíaca. Para classificação usou-se o ponto de corte proposto pela OMS e do National Institutes of Health - NIH (2000) de acordo com o risco de complicações metabólicas associadas à obesidade ${ }^{8}$.

Para avaliação do conhecimento nutricional foi aplicado o Questionário de Conhecimento Nutricional (QCN), desenvolvido por Harnack et al. ${ }^{9}$, adaptado e validado para o Brasil por Scagliusi et al. ${ }^{5} \mathrm{O}$ QCN contém doze tópicos pertinentes ao padrão dietético americano que foi validado para a população brasileira de acordo com a pirâmide alimentar adaptada para o Brasil. Os critérios para classificação da pontuação são: pontuações totais entre zero e seis indicam baixo conhecimento nutricional, entre 


\section{artigo}

Silveira, M.S.; Oliveira, T.M.; Oliveira, A.S.; Santos, D.T.S.; Menezes, R.R.; Lima, M.L.

Conhecimento nutricional entre mulheres Obesas e Eutróficas atendidas em uma unidade ambulatorial de saúde: Classificação e Comparação entre os Grupos

sete e dez indicam moderado conhecimento nutricional e acima de dez indicam alto conhecimento nutricional. ${ }^{9,11}$

Para medir o nível de atividade física foi utilizado o Questionário Internacional de Atividade Física (IPAQ), em sua versão curta, cujo mesmo teve sua validade testada no Brasil por Matsudo et $\mathrm{a}^{10}$. Para fins de análises as variações de "ativo" foram consideradas em uma única variável, e da mesma maneira foi feita com o grupo de insuficientemente Ativo.

Os questionários foram entregues as participantes da pesquisa com orientação verbal e escrita sobre os procedimentos para o preenchimento. Dúvidas foram esclarecidas pelos responsáveis à aplicação do IPAQ e QCN. Não houve comunicação entre os sujeitos da pesquisa durante o preenchimento assim como não se determinou limite de tempo para preenchimento dos formulários. Durante a devolução foram realizadas conferências e na ausência de respostas a participante era orientada a preencher.

Para descrever as variáveis deste estudo, foram utilizadas medidas de tendência central (média) e mediana para as variá- veis contínuas e percentuais para as variáveis categóricas. A normalidade dos dados foi testada por meio do teste Kolmogorov-Smirnov. Associação das variáveis categóricas entre os grupos analisados foram testadas pelo Teste qui-quadrado, exato de Fisher e Linear-by-Linear. A comparação das variáveis contínuas entre grupos foi feita por meio do Teste T student. O nível crítico para determinação do $p$ valor foi de $5 \%$. E todas as análises foram realizadas no programa Statistical Package for the Social Sciences (SPSS) versão 24.

\section{RESULTADOS}

A amostra foi composta por $128 \mathrm{mu}-$ lheres, com idade média de 40,26 anos (DP $\pm 10,29)$ subdivididas proporcionalmente e propositalmente em dois grupos: 64 Mulheres Obesas (MO) e 64 Mulheres Eutróficas (ME). As características dos dois grupos estão apresentadas na Tabela 1. No grupo das MO observou-se a idade média de 45,17 anos (DP $\pm 11,6)$ e $\mathrm{ME}$ com idade média de 35,36 anos (DP \pm $7,9)$. Entre as $\mathrm{MO}$ a maioria eram adultas entre 40 a 60 anos (33\%) e entre as ME a maioria eram jovens adultos de 20 a 40 anos (46\%). Quanto ao estado civil 32\% das $\mathrm{MO}$ eram casadas, enquanto 33\% das ME são solteiras. Já em relação ao nível de conhecimento geral e atividade física, observa-se que a maioria das mulheres classificadas como "baixo nível" são solteiras (63,6\%), como apresentado na Tabela 1.

Ainda referente às variáveis sociodemográficas, 50\% das MO se autodeclaram pretas, enquanto entre as $\mathrm{ME}$ a maioria se declarou não preta (51\%). Em relação ao vínculo empregatício, ambos os grupos se encontram sem vínculo (MO 76,6\% e ME 53,1\%). Sobre a renda familiar, ambos os grupos apresentaram renda familiar de 1 a 2 salários mínimos $(56,3 \%$ do $\mathrm{MO}$ e $67,2 \%$ do $\mathrm{ME}$, com $\mathrm{p}=0,01)$. E entre os grupos, a maioria das ME apresentaram ensino médio completo $(71,9 \%$ com $\mathrm{p}=0,02$ ), assim como as mulheres com nível de conhecimento de "moderado/alto" (67\%), conforme Tabela 1.

Sobre os dados clínicos, a maioria das ME informou ausência de DCNT $(78,1 \%)$ em relação ao $\mathrm{MO}(35,9 \%), \mathrm{p}=$ 0,00 . Já quanto ao nível de conhecimento, o grupo com conhecimento "moderado/

Tabela 1. Características sociodemográficas, de estilo de vida e clínicas de mulheres obesas (MO) e eutróficas (ME): comparação entre os grupos MO e ME e associação entre nível de conhecimento geral e atividade física.

\begin{tabular}{|c|c|c|c|c|c|c|}
\hline Variáveis & $\begin{array}{l}\text { Obesas } n= \\
64(50 \%)\end{array}$ & $\begin{array}{c}\text { Eutróficas } n= \\
64(50 \%)\end{array}$ & P Valor & $\begin{array}{c}\text { Baixo } n=22 \\
(17,2 \%)\end{array}$ & $\begin{array}{c}\text { Mod./ Alto } n= \\
106(82,8 \%)\end{array}$ & P Valor \\
\hline Idade Média (DP) & $\begin{array}{c}45,17( \pm \\
11,6)\end{array}$ & $35,36( \pm 7,9)$ & & $42,3( \pm 11,5)$ & $38,9( \pm 10,9)$ & \\
\hline Adultos Jovens $(20-40)$ & $24(37,5)$ & $46(71,9)$ & & $8(36,3)$ & $62(58,5)$ & \\
\hline Adultos (40-60) & $33(51,5)$ & $18(28,1)$ & $0,00^{*}$ & $13(59,1)$ & $38(35,9)$ & $0,13^{*}$ \\
\hline Idosos (>60) & $7(10,9)$ & 0 & & $1(4,5)$ & $6(5,7)$ & \\
\hline \multicolumn{7}{|l|}{ Estado Civil } \\
\hline Solteira & $31(48,4)$ & $33(51,6)$ & \multirow{2}{*}{$0,74^{*}$} & $14(63,6)$ & $51(48,1)$ & \multirow{2}{*}{$0,24^{*}$} \\
\hline Casada & $33(51,6)$ & $31(48,4)$ & & $8(36,4)$ & $55(51,9)$ & \\
\hline \multicolumn{7}{|l|}{ Raça } \\
\hline Preta & $32(50)$ & $31(48,4)$ & \multirow{2}{*}{$0,86^{*}$} & $14(63,6)$ & $49(46,2)$ & \multirow{2}{*}{$0,13^{*}$} \\
\hline Não Preta (Branca e Parda) & $32(50)$ & $33(51,6)$ & & $8(36,4)$ & $57(53,8)$ & \\
\hline Situação Empregatícia & & & & & & \\
\hline
\end{tabular}




\begin{tabular}{|c|c|c|c|c|c|c|}
\hline Sem vínculo & $49(76,6)$ & $34(53,1)$ & \multirow{2}{*}{$0,00^{* *}$} & 18(81,8) & $65(61,3)$ & \multirow{2}{*}{$0,08^{* *}$} \\
\hline Com vínculo & $15(23,4)$ & $30(46,9)$ & & $4(18,2)$ & $41(38,7)$ & \\
\hline \multicolumn{7}{|l|}{ Renda Familiar } \\
\hline Até 1 Salário & $26(40,6)$ & $11(17,2)$ & \multirow{3}{*}{$0,01^{*}$} & $10(45,5)$ & $27(25,5)$ & \multirow{3}{*}{$0,023^{* *}$} \\
\hline 1 - 2 Salários & $36(56,3)$ & $43(67,2)$ & & $12(54,5)$ & $67(63,2)$ & \\
\hline 2 - 3 Salários & $2(3,1)$ & $10(15,6)$ & & 0 & $12(11,3)$ & \\
\hline \multicolumn{7}{|l|}{ Escolaridade } \\
\hline Fundamental Completo & $24(37,5)$ & $13(20,3)$ & \multirow{3}{*}{$0,22^{*}$} & $9(40,9)$ & $28(26,4)$ & \multirow{3}{*}{$0,95^{* *}$} \\
\hline Médio Completo & $38(59,4)$ & $46(71,9)$ & & $13(59,1)$ & $71(67,0)$ & \\
\hline Superior & $2(3,1)$ & $5(7,8)$ & & 0 & $7(6,6)$ & \\
\hline \multicolumn{7}{|l|}{ DCNT } \\
\hline Sim & $41(64,1)$ & $14(21,9)$ & \multirow{5}{*}{$0,00^{*}$} & $8(36,4)$ & $47(44,3)$ & \multirow{5}{*}{$0,49^{*}$} \\
\hline DM & $20(31,3)$ & $7(11,0)$ & & - & - & \\
\hline HAS & $18(28,1)$ & $5(7,9)$ & & - & - & \\
\hline Outras & $3(4,7)$ & $2(3,0)$ & & - & - & \\
\hline Sem DCNT & $23(35,9)$ & $50(78,1)$ & & $14(63,6)$ & $59(55,7)$ & \\
\hline \multicolumn{7}{|l|}{ Estilo de Vida Tabagista } \\
\hline Sim & $3(4,7)$ & $2(3,1)$ & \multirow{2}{*}{$1,00^{* *}$} & - & $5(4,7)$ & \multirow{2}{*}{$0,587^{* *}$} \\
\hline Não & $61(95,3)$ & $62(96,1)$ & & $22(100)$ & $101(95,3)$ & \\
\hline \multicolumn{7}{|l|}{ Etilista } \\
\hline Sim & $29(45,3)$ & $36(56,2)$ & \multirow{2}{*}{$0,289^{* *}$} & $6(27,3)$ & $59(55,7)$ & \multirow{2}{*}{$0,016^{* *}$} \\
\hline Não & $35(54,7)$ & $28(43,8)$ & & $16(72,7)$ & $47(44,3)$ & \\
\hline
\end{tabular}

Figura 1. Classificação do nível de conhecimento nutricional de acordo com QCN.

Nivel de Conhecimento Nutricional

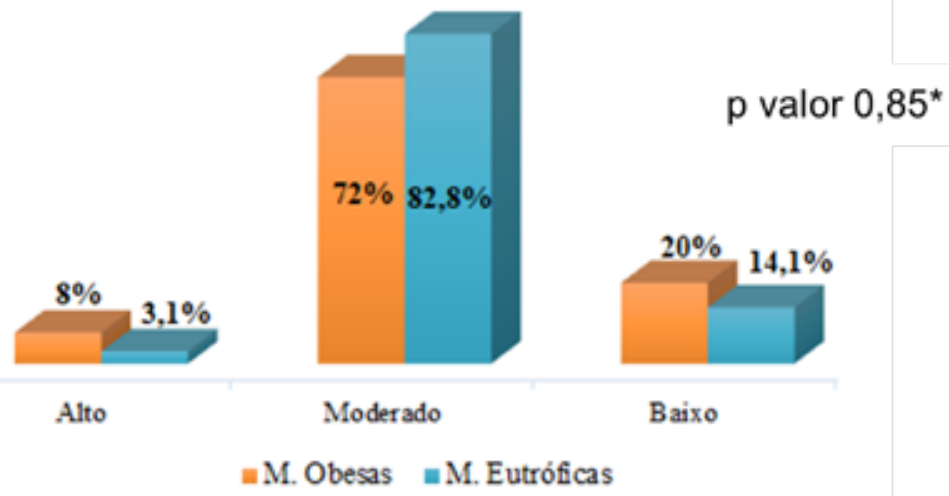

alto", também demonstrou ter ausência das DCNT (55,7\%).

Quanto ao estilo de vida, evidenciou-se uma homogeneidade entre os grupos, que indicou ser não tabagista (MO 95,3\% e ME $96,1 \%$ ), enquanto $56,3 \%$ do grupo das ME relatou ser etilista. Também o grupo de mulheres com nível de conhecimento "moderado/alto" demonstrou maioria ser não tabagista (MO 100\% e ME 95,3\%), porém com predomínio de $55,7 \%$ de ser etilista. Conforme Tabela 1.

Quanto ao Nível de Conhecimento Nutricional (NCN), é possível observar que ambos os grupos apresentaram como maioria a classificação de "moderado" (MO 72\% e ME 82,8\%). Porém, em relação à classificação "alto" e "baixo" destaca-se maioria MO com $8 \%$ e $20 \%$, respectivamente (Figura 1). Não houve significância estatísticas entre os grupos avaliados. 


\section{artigo}

Silveira, M.S.; Oliveira, T.M.; Oliveira, A.S.; Santos, D.T.S.; Menezes, R.R.; Lima, M.L.

Conhecimento nutricional entre mulheres Obesas e Eutróficas atendidas em uma unidade ambulatorial de saúde: Classificação e Comparação entre os Grupos

\section{Tabela 2 - Classificação do nível de atividade física de acordo com IPAQ*}

\begin{tabular}{|c|c|c|c|c|c|c|}
\hline $\begin{array}{c}\text { Classificação de Atividade } \\
\text { Física }\end{array}$ & $\begin{array}{c}\text { Obesasn }=64 \\
(50 \%)\end{array}$ & $\begin{array}{c}\text { Eutróficasn= } \\
64(50 \%)\end{array}$ & p valor & $\begin{array}{c}\text { Baixo } n=22 \\
(17,2 \%)\end{array}$ & $\begin{array}{c}\text { Mod./Alto n= } \\
106(82,8 \%)\end{array}$ & P valor \\
\hline Sedentário & 0 & $2(3,1)$ & - & $2(9,1)$ & 0 & - \\
\hline Ativo & $38(59,4)$ & $45(70,3)$ & - & $12(54,5)$ & $71(67)$ & - \\
\hline
\end{tabular}

Tabela 3 - Associação do Nível de Conhecimento Nutricional e variáveis antropométricas.

Variáveis

Nível de Conhecimento Nutricional

$\begin{array}{lccc} & \text { Baixo X (SD) } \mathbf{n}=22 & \text { Mod./ Alto X (SD) } \mathbf{n}=106 & \text { P valor } \\ \text { CC (cm) } & 91,8(17,1) & 89,9(17,6) & 0,651 * \\ \text { IMC (Kg/m²) } & 32,4(9,1) & 29,7(8,9) & 0,221^{*} \\ \text { * Teste Tstudent } & & & \end{array}$

Quanto ao nível de atividade física destaca-se que a maioria dos grupos apresentaram a classificação" ativo" (MO 59,4\% e ME 70,3\%). E quando comparadas ao nível de conhecimento, as mulheres classificadas como "Baixo" apresentaram classificação de "ativo" (4,5\%), enquanto as classificadas como "Mod./Alto" apresentaram a classificação como "insuficientemente ativo" (93,4\%), como descrito na Tabela 2.

Também foi possível associar o NCN com algumas variáveis, como descrito na Tabela 3, sendo observado que não houve diferença estatística entre as variáveis.

\section{DISCUSSÃO}

O presente estudo foi realizado com uma amostra total de 128 mulheres, subdivididas igualmente em dois grupos (64 MO e $64 \mathrm{ME}$ ), atendidas no Centro Médico da Baiana, vinculado à EBMSP, na cidade de Salvador-Bahia. De acordo com a Política Nacional de Atenção Integral à Saúde da Mulher ${ }^{11}$, as mulheres são as principais usuárias do SUS, frequentam os serviços de saúde acompanhando crianças e outros familiares, mas sobretudo buscam o seu próprio atendimento para prevenção e tratamento de doenças.

De uma maneira global a amostra foi composta predominantemente por mulheres em fase adulta jovens, solteiras e au- todeclaradas pretas. A maioria não possuía vínculo empregatício, tinham renda familiar entre 1-2 salários mínimos e possuíam ensino médio completo. Esses dados são reforçados em literatura, visto que a maior parte da população brasileira advém de famílias de classe baixa à média, onde predomina a raça de pretos e pardos. Informações essas, que ficam ainda mais evidentes quando comparado às regiōes do Brasil, em que se destaca a região nordeste com maior prevalência. ${ }^{12,13}$

Em relação à idade, as mulheres eutróficas eram mais jovens enquanto as obesas estavam em maior quantidade na faixa etária de 40 a 60 anos e essa diferença foi estatisticamente significante entre os grupos. Esses dados eram esperados considerando que com o aumento da idade e as alterações hormonais, as mulheres tendem a ganhar mais peso. ${ }^{14}$

Sobre os aspectos clínicos, observou-se que a maioria das $\mathrm{MO}$ referiram algum tipo de DCNT, enquanto no grupo de ME a maioria indicou ausência de doenças desse gênero. Já é consenso na literatura as consequências geradas pela obesidade no organismo, uma vez que representa um dos fatores determinantes para a resistência à insulina e o surgimento de doenças cardiovasculares. ${ }^{13}$

A prevalência de DCNTs também é maior em populações mais vulneráveis socioeconomicamente, por estarem mais expostas aos fatores determinantes e condicionantes. Dentre esses fatores são descritos na literatura o acesso limitado e a baixa procura aos serviços de saúde ligados à promoção de uma alimentação saudável adequada que possa prevenir doenças crônicas não transmissíveis. ${ }^{14,15,16}$ Já quando relacionado ao nível de atividade física, se percebe-se que há predominância da ausência de DCNT, corroborando com estudos que elucidam que quanto maior a prática da atividade física maior a prevenção contra essas doenças. ${ }^{17,18}$

Quanto ao estilo de vida, verificou-se que ambos os grupos informaram não ser tabagistas, enquanto a maioria das $\mathrm{ME}$ declararam ser etilistas. Este resultado reforça dados da literatura e é comumente justificado pela mudança socioeconômica, epidemiológica e nutricional que o país vem sofrendo nas últimas décadas, mais expressivo sobretudo nas mulheres. De acordo com dados da VIGITEL, houve um aumento nos índices de ingestão alcoólica em mulheres brasileiras nos anos de 2006 a 2018.20 Mundialmente, estudos trazem um aumento no consumo de bebidas alcoólicas por mulheres, além disso, maiores taxas de mortalidade vêm sendo documentadas relacionada ao uso

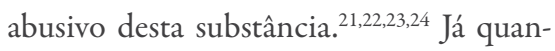
to ao nível de atividade física, verifica-se que em sua maioria as mulheres de ambos os grupos apresentaram a classificação de “ativo". Atualmente, observa-se uma crescente busca pela prática de atividades físicas. Segundo Machado e colaboradores $(2013)^{25}$, a prática de atividade física aliada à intervenção nutricional e ao aumento do conhecimento em nutrição resulta em um melhor consumo alimentar e con- 
sequentemente mudanças positivas em parâmetros antropométricos, além de prevenir o desenvolvimento de doenças crônicas. ${ }^{11,19,25}$ Quando comparadas o nível de conhecimento e de atividade física, destacou-se que as classificadas como "Mod./ Alto" nível de conhecimento obtiveram a classificação de "insuficientemente ativo" em relação à atividade física. De acordo com a literatura, estes achados podem ser justificados pela rotina dessas mulheres, que acabam não tendo um planejamento para realização destas atividades. ${ }^{19,25,27}$

Neste estudo, apenas os dados de renda e etilismo estiveram associados significativamente ao um melhor nível de conhecimento nutricional. Em relação à escolaridade houve diferença entre os grupos, porém sem relevância estatística. Esse resultado contrasta com achados na literatura que demonstram associações positivas entre nível de conhecimento e escolaridade, evidenciando a importância da educação escolar como fator básico para obtenção de conhecimento relacionados à nutriçãao ${ }^{28,29,30,31}$.

A partir dos achados no presente estudo, podemos considerar que as variáveis sociodemográficas e econômicas agem como fator influente e geram impacto sobre o NCN, bem como na prática de atividades físicas. Silva e colaboradores (2016) 32 reforçam que essas condições geram impactos sobre cuidado com a saúde e alimentação, afetando a qualidade de vida e estilo de vida, mas não só dessas mulheres, como de suas famílias.

Ainda sobre o nível de conhecimento nutricional, pôde-se observar que em ambos os grupos (MO e ME) houve a

\section{Foi possível também verificar que não}

houve associação

estatisticamente

significante

entre o NCN e

algumas variáveis

antropométricas

(CC e IMC).

predominância da classificação de um conhecimento "moderado". Em literatura, informações acerca do nível de conhecimento nutricional ainda é escasso, em especial na população estudada. Entretanto, é possível verificar que a partir da aplicação de outros questionários que avaliam esta mesma variável, os resultados se assemelham, indicando sempre em sua maioria uma classificação como "médio" ou "moderado". Sendo assim, corroboram com os achados do presente estudo, que indicam um nível de conhecimento moderado ou intermediário. ${ }^{12,18,27}$

Foi possível também verificar que não houve associação estatisticamente significante entre o $\mathrm{NCN}$ e algumas variáveis antropométricas (CC e IMC). Sobre isso, um estudo desenvolvido por O' Brien e Devies $^{32}$, com 500 adultos em sua maioria mulheres com idade média de 41,9 anos na Irlanda do Norte utilizando questionário geral de nutrição, observaram que o alto nível de conhecimento nutricional não se correlacionou com IMC, destacando que esse pode não ser um fator determinante para indivíduos adotarem hábitos alimentares saudáveis. Outros estudos corroboraram com os resultados, confirmando que dados antropométricos como IMC e CC também não estão associados ao nível de conhecimento nutricional ${ }^{13,33,34,35,36}$.

Como limitação do estudo, considerou-se tamanho da amostra, entretanto existe a necessidade de obter cada vez mais a compreensão dos fatores que se interrelacionam ao NCN.

\section{CONCLUSÃO}

A maioria do grupo de obesas e eutróficas apresentou um nível de conhecimento nutricional "moderado", sendo este conhecimento associado a maior renda familiar (ainda que com renda familiar entre 1 e 2 salários mínimos), ao maior consumo de bebida alcoólica e indivíduos mais ativos. $\mathrm{O}$ conhecimento dos fatores interligados ao nível de conhecimento nutricional é necessário para possibilitar a adoção de ferramentas que auxiliem em ações educativas, melhor preparo aos profissionais de saúde envolvidos e por fim planejar estratégias de saúde pública que possam auxiliar na melhora do hábito alimentar e consequentemente prevenir ocorrência de DCNT.

\section{REFERÊNCIAS}

1. Leonell, Amália et al. Comida, ato alimentar e outras reflexões consumidas. Contextos da Alimentação-Revista de Comportamento, Cultura e Sociedade, 2017; 5(2):3-13p.

2. Júnior, Cláudio Santiago Dias et al. Excesso de peso, obesidade e educação no Brasil. Saúde (Santa Maria), 2019; 45(2).

3. Assumpção, Daniela de et al. Diferenças entre homens e mulheres na qualidade da dieta: estudo de base populacional em Campinas, São Paulo. Ciência \& Saúde Coletiva, 2017; 22: 347$358 p$.
4. Axelson M, Brinberg D. The measurement and conceptualization of nutrition knowledge. Journal Nutrition Education,1992; 24(5):239-246p.

5. Scagliusi F. B, Polacow VO, Cordas TA, Coelho D, Alvarenga M, Philippi ST. Tradução, adaptação e avaliação psicométrica da Escala de Conhecimento Nutricional do National Interview Survey Cancer Epidemiology. Revista de Nutrição, 2006; 19(4): 425436p.

6. Pereira, Mariana Milani et al. Influência do ambiente familiar 


\section{REFERÊNCIAS}

no desenvolvimento do comportamento alimentar. Revista Uningá, 2014; 41 (1).

7. Tavares, Helder Cardoso et al. A importância da inserção do nutricionista na Unidade Básica de Saúde: percepção dos profissionais de saúde. Revista e-ciência, 2013; 4(1):. 89-98p. .

8. National Institutes of Health, NIH. The practical guide identification, evaluation, and treatment of overwheight and obesity in adutlts. Bethesda, MD: 2000.

9. Harnack L, Block G, Subar A, Lane S, Brand R. Association of cancer prevention-related nutrition knowledge, beliefs, and attitudes to cancer prevention dietary behavior. Journal of the American Dietetic Association. 1997; 97 (9): 957-965.

10. Matsudo SM, Araújo TL, Matsudo VKR, Andrade DR, Andrade EL, Oliveira LC, et al. Questionário Internacional de Atividade Física (IPAQ): estudo de validade e reprodutibilidade no Brasil. Rev Bras Ativ Saude. 2001.

11. BRASIL. Ministério da Saúde. Secretaria de Atenção à Saúde. Departamento de Ações Programáticas Estratégicas. Política nacional de atenção integral à saúde da mulher: princípios e diretrizes / Ministério da Saúde, Secretaria de Atenção à Saúde, Departamento de Ações Programáticas Estratégicas. - Brasília: Ministério da Saúde, 2012.

12. Fundação Oswaldo Cruz (Fiocruz). Vozes da Classe Média: É ouvindo a população que se constroem políticas públicas adequadas. Brasília, $1^{a}$ edição. 2012. 52p.

13. Castro NMG, Dáttilo M e Lopes LC. Avaliação do conhecimento nutricional de mulheres fisicamente ativas e sua associação com o estado nutricional. Rev. Bras. Cienc. Esporte, Campinas. 2010. 32 (1): 161-172p.

14. Bernal RTI, Felisbino-Mendes MS, Carvalho QH, Pell J, Dundas $R$, Leyland $A$, et al. Indicators of chronic noncommunicable diseases in women of reproductive age that are beneficiaries and non-beneficiaries of Bolsa Família. Rev Bras Epidemiol. 2019; 22(2):13p.

15. Gallon CW e Wender MCO. Nutritional status and quality of life of climacteric women. Rev Bras Ginecol Obstet. 2012; 34(4):175-83p.

16. Leão EM e Marinho LFB. Saúde das mulheres no Brasil: subsídios para as políticas públicas de saúde. Ministério da SaúdeMS: Promoção à Saúde. 2000; 31-36p.

17. Vasques ACJ, Priore SE, Rosado LEFPL e Franceschini SCC. The use of anthropometric measures to assess visceral fat accumulation. Rev. Nutr., Campinas, 2010; 23(1):107-118p.

18. Abegunde DO, Mathers CD, Adam T, Ortegon M e Strong K.The burden and costs of chronic diseases in low-income and middle-income countries. The Lancet, 2010; 370(9603):1929$38 p$

19. Fang X, Han D, Cheng Q, Zhang P, Zhao C, Min J, et al. Association of Levels of Physical Activity With Risk of Parkinson Disease A Systematic Review and Meta-analysis. JAMA Netw Open. 2018; 1(5): e182421.
20. Brasil. Ministério da Saúde. Secretaria de Vigilância em Saúde. Departamento de Análise em Saúde e Vigilância de Doenças não Transmissíveis.VIGITEL 2018: Vigilância de fatores de risco e proteção para doenças crônicas por inquérito telefônico. Departamento de Análise em Saúde e Vigilância de Doenças não Transmissíveis. - Brasília: Ministério da Saúde, 2019.

21. Anishchenko AP, Arkhangel'skaya AN, Pustovalov DA, Rogoznaya EV, Urakov AL e Gurevich KG. [The association between physical inactivity and other behavioural risk factors of the development of chronic non-communicative diseases among the students]. opr Kurortol Fizioter Lech Fiz Kult. 2017;94(1):15-20p.

22. Marcacine PR, Castro SS, De Castro SS, Meirelles MCCC, Haas VJ e Walsh IAP. Quality of life, sociodemographic and occupational factors of working women.Ciência \& Saúde Coletiva, 2019; 24(3):749-760p.

23. Chaieb JA e Castellarin C. Association between smoking and alcoholism: initiation into the major human dependencies. Rev. Saúde Pública, 1998; 32 (3):246-254p.

24. Rehm J, Baliunas D, Borges GLG, Graham K, Irving H, Kehoe T, et al. The relation between different dimensions of alcohol consumption and burden of disease: an overview. NIH Public Access, 2010; 105(5):817-843p.

25. Barbosa LB, Vasconcelos SML, Correia LOS e Ferreira RC. Nutrition knowledge assessment studies in adults: a systematic review. Ciência \& Saúde Coletiva, 2016; 21(2):449-462p.

26. Machado et al. Efetividade de uma intervenção nutricional associada à prática de atividade física Cad. Saúde Colet, 2013; 21 (2): 148-53p

26. World Health Organization (WHO). Global status report on alcohol and health 2014. WHO Library Cataloguing-in-Publication Data, 2014. 392p.

27. Lin W, Lee YW. Nutrition knowledge, attitudes and dietary restriction behaviour of Taiwanese elderly. Asia Pac J Clin Nutr. 2005; 14 (3):221-229p.

28. Nuss H, Freeland-graves J, Clarke K, Klohe-lehman D, Milani TJ. Greater Nutrition Knowledge Is Associated with Lower 1-Year Postpartum Weight Retention in Low-Income Women. J Am Diet Assoc.

29. De Vriendt T, Matthys C, Verbeke W, Pynaert I, De Henauw S. Determinants of nutrition knowledge in young and middle-aged Belgian women and the association with their dietary behaviour. Appetite. 2009; 52 (3):788-792.

30. Hendrie G, Coveney J, Cox D. Exploring nutrition knowledge and the demographic variation in knowledge levels in an Australian community sample. Public Health Nutr. 2008; 11, 1365$1371 p$.

32. Klotz-Silva J, Prado SD e Seixas CM.Eating behavior in the field of Food and Nutrition: what are we talking about? Physis, 2016; 26(4): 1103-23p.

33. O'brien G, Davies M. Nutrition knowledge and body mass index. Health Education Research, 2007; 22 (4): 571-755p. 